Estudo Químico e Avaliação Biológica da Própolis Vermelha de Alagoas

\author{
Aguiar, G. R.; de Lemos, T. L. G.; ${ }^{*}$ Dornelas, C. A.; da Silva, A. M.; de \\ Almeida, M. C. S.; Ferreira, D. A.; Monte, F. J. Q.; Braz-Filho, R.; de \\ Oliveira, I. R.; do Nascimento, P. G. G.
}

Rev. Virtual Quim., 2018, 10 (1), 2-12. Data de publicação na Web: 19 de janeiro de 2018

http://rvq.sbq.org.br

\title{
Chemical Study and Biological Evaluation of the Red Propolis from Alagoas
}

Abstract: Chemical investigation of the ethanol extract of propolis allowed the isolation of 2'-hydroxy4',7-dimethoxyisoflavan (1) 2',7-dihydroxy-4'-methoxyisoflavan (2) 2',4'-dihydroxy-7-methoxyisoflavan (3), 4',7-dihydroxy-2'-methoxyisoflavan (4) 2',4',4-trihydroxychalcone (5) and lup-20(29)-en-3-ol (6). The structures of compounds were identified based on their spectral data and by comparison with the literature. It was performed test to evaluate antioxidant activity by the 2,2-diphenyl-1-picrylhydrazyl (DPPH) method of the ethanol extract and its fractions obtaining significant results similar to Vitamin C. It was also evaluated anticholinesterase activity, observing promising results with the extract and fractions. Being the red propolis of these activities, it can be used in the treatment of degenerative diseases such as Alzheimer. There are already records of propolis as an antioxidant, however, the activity of anticholinesterase is being cited for the first time.

Keywords: Red propolis; antioxidant activity; anticholinesterase activity.

\section{Resumo}

A investigação química do extrato etanólico de própolis vermelha permitiu o isolamento de: $2^{\prime}$-hidroxi4',7-dimetoxiisoflavana (1), 2',7-dihidroxi-4'-metoxiisoflavana (2), 2', 4'-dihidroxi-7-metoxiisoflavana (3), 4',7-dihidroxi-2'-metoxiisoflavana (4), 2',4',4-trihidroxichalcona (5) e lup-20(29)-en-3-ol (6). As estruturas das substâncias foram caracterizadas com base nos seus dados espectrais e por comparação com a literatura. Foi realizado o teste de atividade antioxidante pelo método do 2,2-difenil-1picrilhidrazil (DPPH) do extrato etanólico e suas frações, obtendo-se resultados significativos e semelhantes ao padrão Vitamina C. Também foi avaliada atividade anticolinesterásica, observando-se resultados promissores com o extrato e frações. Sendo a própolis vermelha possuidora destas atividades, esta pode ser usada como auxiliar em doenças degenerativas como Alzheimer. Já existem registros de própolis como agente antioxidante, entretanto, a atividade anticolinesterásica está sendo citada pela primeira vez.

Palavras-chave: Própolis vermelha; atividade antioxidante; atividade anticolinesterásica.

* Universidade Federal do Ceará, Departamento de Química Orgânica e Inorgânica, Campus do Pici, CEP 60021-940, Fortaleza-CE, Brasil..

Mtlemos@dqoi.ufc.br

DOI: 10.21577/1984-6835.20180002

Rev. Virtual Quim. |Vol 10| | No. 1| |2-12| 


\section{Estudo Químico e Avaliação Biológica da Própolis Vermelha de Alagoas}

Gisele R. Aguiar, ${ }^{a}$ Telma L. G. de Lemos, ${ }^{a}, *$ Conceição A. Dornelas, ${ }^{b}$ André M. da Silva, ${ }^{a}$ Macia C. S. de Almeida, ${ }^{a}$ Daniele A. Ferreira, ${ }^{a}$ Francisco J. Q. Monte, ${ }^{\mathrm{a}}$ Raimundo Braz-Filho, ${ }^{\mathrm{c}}$ Irvila R. de Oliveira, ${ }^{\mathrm{a}}$ Patrícia Georgina G. do Nascimento ${ }^{a}$

a Universidade Federal do Ceará, Departamento de Química Orgânica e Inorgânica, Campus do Pici, CEP 60451-970, Fortaleza-CE, Brasil.

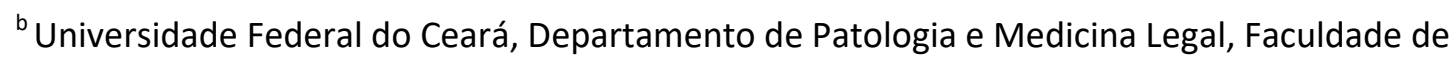
Medicina, CEP 60441-750, Fortaleza-CE, Brasil.

${ }^{\text {C } P e s q u i s a d o r ~ E m e ́ r i t o ~-~ F A P E R J / D e p a r t a m e n t o ~ d e ~ Q u i ́ m i c a ~-~ I C E ~-~ U F R R J / L a b o r a t o ́ r i o ~ d e ~}$ Ciências Químicas - CCT - UENF, Av. Alberto Lamego, N² 2000, CEP 28013-600, Campos dos Goytacazes-RJ, Brasil.

*tlemos@dqoi.ufc.br

Recebido em 24 de março de 2017. Aceito para publicação em 16 de janeiro de 2017

\section{Introdução}

\section{Metodologia}

2.1. Coleta do material vegetal

2.2. Obtenção do extrato etanólico da própolis vermelha

2.3. Isolamento dos constituintes da própolis vermelha

2.4. Dados físicos dos metabólitos secundários isolados

2.5. Teste de atividade antioxidante

2.6. Ensaio para inibição da enzima acetilcolinesterase

\section{Resultados e Discussão}

4. Considerações Finais

\section{Introdução}

A própolis, material resinoso coletado e processado pelas abelhas a partir de exsudatos de brotos e botões florais de várias plantas, ${ }^{1}$ possui colorações que variam do verde, vermelho a marrom, odor característico e também propriedades adesivas por conta de sua forte interação com óleos e proteínas da pele. ${ }^{2}$ É utilizada pelas abelhas para manutenção $d a$ temperatura interna, reparo dos favos de mel, embalsamar insetos mortos e proteger a colmeia contra a invasão de microrganismos. ${ }^{1}$

Cerca de 300 compostos foram 
identificados até o momento de diferentes amostras de própolis. ${ }^{3}$ A composição química da própolis está relacionada com a biodiversidade da região em que foi originada e com a época de sua coleta e, em geral, consistem em ceras, resinas, compostos inorgânicos, óleos essenciais, compostos fenólicos, ácidos e ésteres alifáticos, ácidos e ésteres aromáticos, ácidos graxos, chalconas, terpenoides, esteroides, álcoois, proteínas, vitaminas $\mathrm{B} 1, \mathrm{~B} 2, \mathrm{~B} 6, \mathrm{C}$ e E. ${ }^{2}$

Há séculos tem sido usada empiricamente como remédio tradicional na medicina popular, sendo conhecido por seus benefícios potenciais para a saúde humana e por apresentar diversas atividades biológicas, tais como: citotóxica, imunomodulatória, antiviral, antioxidante, antitumoral, antimicrobiana e anti-inflamatória. ${ }^{2,4,5,6}$

A própolis tem sido comercializada por indústrias farmacêuticas como medicina alternativa para prevenir doenças, tais como diabetes, doenças cardíacas e até mesmo o câncer. ${ }^{7}$

O Brasil é considerado um dos maiores fornecedores de própolis do mundo. Os diferentes tipos de própolis brasileira aparecem relatados com base na composição química e propriedades biológicas distintas quando coletadas em regiões diferentes do país. Em razão da grande diversidade da flora brasileira, as própolis identificadas no Brasil foram classificadas de acordo com sua origem geográfica, composição química, origem vegetal e atividades biológicas em 12 grupos diferentes: um da Região Sudeste, cinco da Região Sul e seis no Nordeste. ${ }^{1,2}$

Recentemente um tipo novo de própolis proveniente da região Nordeste do país foi denominada de "própolis vermelha" e classificada como sendo o 13 tipo, que revelou composição química rica em isoflavonoides. Sua origem botânica foi identificada como Dalbergia ecastophyllum, uma leguminosa proveniente da região do mangue do estado de Alagoas, rica em flavonoides. Esta espécie é relatada como sendo proveniente de Cuba e Venezuela, originada das plantas Clusia nemorosa e
Clusia scrobiculata, respectivamente, família Clusiaceae. $^{2,8}$

Estudos recentes mostraram que a própolis vermelha apresentou quantidades elevadas de isoflavonas em grupo particular de flavonoides que apresentam inúmeras propriedades biológicas, dentre as quais se destacam antiviral, antimicrobiana, antiinflamatória, anticancerígena e antiulcerativa. ${ }^{3,6,8}$

O presente trabalho relata o isolamento e determinação estrutural dos constituintes químicos do extrato etanólico da própolis vermelha de Alagoas, bem como a avaliação da atividade antioxidante e anticolinesterásica do extrato e suas frações.

\section{Metodologia}

\subsection{Coleta do material vegetal}

O material foi coletado de uma amostra de própolis vermelha na cidade de Barra de Santo Antônio - Alagoas, durante o mês de junho de 2006, e em seguida, foi triturado e submetido à extração com etanol a frio.

2.2. Obtenção do extrato etanólico da própolis vermelha

A própolis $(520,0 \mathrm{~g})$ foi triturada, armazenada em reservatório de vidro com tampa, acrescida de etanol 95\% (Vetec ${ }^{\circledR}$ ) e mantida durante duas semanas em repouso para extração. Decorrido esse tempo, o solvente foi destilado em rotaevaporador sob pressão reduzida, fornecendo $100,0 \mathrm{~g}$ de extrato etanólico (EEPV) com rendimento de 19,2\% em relação ao material de partida.

2.3. Isolamento dos constituintes da própolis vermelha

O extrato EEPV $(30,0 \mathrm{~g})$ foi misturado com sílica gel e submetido à coluna filtrante sob 
vácuo, usando como eluentes: hexano, diclorometano, acetato de etila e metanol puros, seguindo uma ordem de polaridade crescente. Em seguida a fração diclorometano $(11,5$ g) foi submetida à coluna filtrante gravitacional, usando como eluentes: hexano, diclorometano, acetato de etila e metanol, puros e em misturas binárias em ordem crescente de polaridade.

A fração FDPV (2,9 g) foi adsorvida em gel de sílica, pulverizada em gral de porcelana e disposta em coluna de vidro com gel de sílica empacotada com hexano. A eluição foi realizada com hexano, acetato de etila, metanol e água, puros ou em misturas binárias em escala crescente de polaridade, obtendo-se 125 frações. Após análise por $C C D$, as frações foram reunidas, e a fração 37-43 (7:3 Hex/AcOEt, 230,0 mg) foi recromatografada em coluna de exclusão com Sephadex LH-20 usando metanol como fase móvel. Depois da análise por CCD, foram reunidas as frações 40-80 (150,0 mg) de acordo com sua semelhança, e em seguida cromatografada em coluna "flash" usando a solução binária hexano/AcOEt $20 \%$. A fração 26-46 (6,0 mg), solúvel em $\mathrm{CH}_{2} \mathrm{Cl}_{2}$, apresentou-se uniforme em $C C D$ e foi identificada como 2'-hidroxi-4',7dimetoxiisoflavana (1).

A fração 7-33 (93,5 mg), proveniente da fração 37-43 (FDPV), foi recromatografada em coluna de gel de sílica usando os solventes: hexano, AcOEt e $\mathrm{MeOH}$ puros ou em misturas binárias de acordo com seu grau de polaridade. Após análise por CCD, foram reunidas as frações 6-8 (9:1 Hex/AcOEt, 11,8 $\mathrm{mg}$ ), um sólido branco amorfo solúvel em $\mathrm{CH}_{2} \mathrm{Cl}_{2}$, identificado como lup-20(29)-en-3-ol (6).

O resíduo da própolis vermelha $(1,1 \mathrm{~g})$ formado durante a extração etanólica foi adsorvido em gel de sílica, pulverizado em gral de porcelana e disposto em coluna de vidro com gel de sílica empacotada com hexano. Foram utilizados os solventes: hexano, AcOEt, MeOH e água puros, ou em misturas binárias em escala crescente de polaridade, obtendo-se 162 frações. Após análise por CCD, as frações semelhantes foram reunidas, e a fração 69-162 (130,6 mg) foi recromatografada em coluna de gel de sílica usando misturas binárias de hexano, AcOEt e $\mathrm{MeOH}$ em grau crescente de polaridade, e fornecendo 139 frações. Após análise em CCD, as frações 29-31 (7:3 Hex/AcOEt, 5,0 mg) foram reunidas de acordo com sua semelhança, solúvel em $\mathrm{CH}_{2} \mathrm{Cl}_{2}$, e identificada como 2',7-dihidroxi-4'metoxiisoflavana (2).

A fração FAEEPV (5,9 g) foi adsorvida em gel de sílica, pulverizada em gral de porcelana e acondicionada em coluna de gel de sílica empacotada com hexano. Os solventes usados como eluentes foram: hexano, AcOEt e $\mathrm{MeOH}$ puros, ou combinados em mistura binária. Após análise por $C C D$, as frações semelhantes foram reunidas e a fração 11 (435,0 mg) foi novamente cromatografada sob média pressão usando uma mistura binária de hexano/AcOEt $40 \%$, e fornecendo 144 frações. Depois de analisadas em CCD, as frações 59-80 (40,0 mg) foram reunidas por apresentarem uma mancha homogênea, solúvel em $\mathrm{MeOH}$, identificada como 4',7dihidroxi-2'-metoxiisoflavana (3).

As frações 117-144 (6:4 Hex/AcOEt, 148,1 $\mathrm{mg})$, provenientes da fração 11 citada acima, foram reunidas e recromatografada em CLAE. Para o desenvolvimento do método $20,0 \mathrm{mg}$ da amostra foi solubilizada em $1 \mathrm{~mL}$ de uma mistura de solventes metanol/água na proporção 6:4 e, em seguida, filtrada em um sistema manual de filtros. Em seguida, $200 \mu \mathrm{L}$ da amostra foi injetada em coluna semipreparativa de fase reversa ODS C18 (250 x 10,0 mm), num sistema isocrático utilizando como fase móvel metanol/água $6: 4$, fluxo de $3 \mathrm{~mL} / \mathrm{min}$ e tempo de corrida de $40 \mathrm{~min}$. Após o desenvolvimento do método, $128,1 \mathrm{mg}$ da amostra foi solubilizada em 6,4 $\mathrm{mL}$ de metanol/água 6:4 e filtrada manualmente. A amostra foi reinjetada nas mesmas condições descritas anteriormente. Foram coletados 6 compostos $(A-F)$, dos quais, o composto F-CLAE foi coletado e concentrado sob pressão reduzida, fornecendo $26,0 \mathrm{mg}$ de um sólido vermelho 
solúvel em $\mathrm{MeOH}$, identificado como 2',4'dihidroxi-7-metoxiisoflavana (4).

Seguindo o mesmo procedimento do isolamento da substância 4, o composto DCLAE foi coletado e concentrado sob pressão reduzida, fornecendo $30,0 \mathrm{mg}$ de um sólido amarelo solúvel em $\mathrm{MeOH}$, identificado como 2', 4',4-trihidroxichalcona (5).

\subsection{Dados físicos dos metabólitos secundários isolados}

\section{2'-hidroxi-4',7-dimetoxiisoflavana}

Sólido rosa; p.f.: $108,2-110,7{ }^{\circ} \mathrm{C}$; IV $\left(\mathrm{cm}^{-1}\right)$ : $\mathrm{V}_{\max } 3374 ; 2917 ; 2843 ; 1618 ; 1506 ; 1444$; $1260 ; 1205 ; 1160 ; 1114 ; 1035$; RMN de ${ }^{13} \mathrm{C}$ (75 MHz, $\left.\mathrm{CDCl}_{3}\right) \delta_{\mathrm{C}}: 30,6\left(\mathrm{CH}_{2}-4\right) ; 32,1(\mathrm{CH}-3)$; 55,6 (C-4'a e C-7a); 70,2 ( $\left.\mathrm{CH}_{2}-2\right) ; 101,7(\mathrm{CH}-$ $\left.3^{\prime}\right) ; 102,4(\mathrm{CH}-8) ; 106,4(\mathrm{CH}-6) ; 107,5$ (CH-5'); 114,7 (C-1'); 120,2 (C-10); 128,5 (CH-6'); 130,4 (CH-5); 154,4 (C-2'); 155,4 (C-9); 159,4 (C-7); 159,7 (C-4'); EM m/z 286, $\mathrm{C}_{17} \mathrm{H}_{18} \mathrm{O}_{4}$.

\section{2',7-dihidroxi-4'-metoxiisoflavana}

(2)

Sólido rosa; IV $\left(\mathrm{cm}^{-1}\right)$ : $\mathrm{v}_{\max } 3346 ; 2924 ; 1619$; $1596 ; 1456 ; 1267 ; 1201 ; 1154 ; 1112 ; 1031$; RMN de ${ }^{13} \mathrm{C}\left(125 \mathrm{MHz}, \mathrm{CDCl}_{3}\right) \delta_{\mathrm{C}}: 30,6\left(\mathrm{CH}_{2}-4\right)$; 31,9 (CH-3); 55,6 (C-4'a); 70,1 ( $\left.\mathrm{CH}_{2}-2\right) ; 102,4$ $\left(\mathrm{CH}-3^{\prime}\right) ; 103,4$ (CH-8); 106,2 (CH-5'); 108,1 (CH-6); 114,9 (C-10); 120,1 (C-1'); 128,4 (CH$\left.6^{\prime}\right) ; 130,6$ (CH-5); 154,5 (C-2'); 155,1 (C-9); 155,4 (C-7); 159,6 (C-4'); EM m/z 272, $\mathrm{C}_{16} \mathrm{H}_{16} \mathrm{O}_{4}$.

\section{4',7-dihidroxi-2'-metoxiisoflavana}

Sólido vermelho; p.f.: $130,3-136,1{ }^{\circ} \mathrm{C}$; IV (cm $\left.{ }^{1}\right)$ : $v_{\max } 3394 ; 2921 ; 2818 ; 1596 ; 1453 ; 1283$; 1203; 1159; 1109; 1027; RMN de ${ }^{13} \mathrm{C}$ (75 $\left.\mathrm{MHz}, \mathrm{CD}_{3} \mathrm{OD}\right) \delta_{\mathrm{C}}: 31,5\left(\mathrm{CH}_{2}-4\right) ; 33,2(\mathrm{CH}-3)$; 55,7 (C-2'a); 71,2 ( $\left.\mathrm{CH}_{2}-2\right) ; 102,6 \quad\left(\mathrm{CH}-3^{\prime}\right)$; 103,9 (CH-8); 105,9 (CH-5); 109,1 (CH-6); 115,1 (C-10); 121,5 (C-1'); 129,0 (CH-6'); 131,4 (CH-5); 156,5 (C-9); 157,3 (C-7); 157,6 (C-4'); 160,9 (C-2'); EM m/z 272, $\mathrm{C}_{16} \mathrm{H}_{16} \mathrm{O}_{4}$.

2',4'-dihidroxi-7-metoxiisoflavana Sólido vermelho; p.f.: $140-143{ }^{\circ} \mathrm{C}$; IV $\left(\mathrm{cm}^{-1}\right)$ : $V_{\max } 3350 ; 2919 ; 1618 ; 1587 ; 1452 ; 1261$; 1198; 1152; 1111; 1029; RMN de ${ }^{13} \mathrm{C}(125$ $\left.\mathrm{MHz}, \mathrm{CD}_{3} \mathrm{OD}\right) \delta_{\mathrm{c}}: 31,5\left(\mathrm{CH}_{2}-4\right) ; 33,2(\mathrm{CH}-3)$; 55,8 (C-7a); 71,4 ( $\left.\mathrm{CH}_{2}-2\right) ; 102,4$ (CH-8); 103,7
$\left(\mathrm{CH}-3^{\prime}\right) ; 107,8$ (CH-5'); 108,0 (CH-6); 116,2 (C10); 120,1 (C-1'); 129,0 (CH-6'); 131,3 (CH-5); 156,6 (C-9); 157,4 (C-2'); 158,1 (C-4'); 160,6 (C-7); $\mathrm{EM} \mathrm{m/z} \mathrm{272,} \mathrm{C}_{16} \mathrm{H}_{16} \mathrm{O}_{4}$.

2',4',4-trihidroxichalcona (5) Sólido amarelo; p.f.: $181,4-183,3{ }^{\circ} \mathrm{C}$; IV $\left(\mathrm{cm}^{-1}\right)$ : $\mathrm{V}_{\max }$ 3284; 1629; 1513; 1226; RMN de ${ }^{13} \mathrm{C}$ (75 $\left.\mathrm{MHz}, \mathrm{CD}_{3} \mathrm{OD}\right) \delta_{\mathrm{C}}: 104,1$ (CH-3'); 109,6 (CH-5'); 114,6 (C-1'); 117,1 (CH-3/5); 118,5 (CH-8); 128,0 (C-1); $131,9(\mathrm{CH}-2 / 6) ; 133,5\left(\mathrm{CH}-6^{\prime}\right)$; 145,6 (CH-7); 161,7 (C-4); 167,2 (C-2'); 167,7 (C-4'); 193,5 (C-9); EM m/z 256, $\mathrm{C}_{15} \mathrm{H}_{12} \mathrm{O}_{4}$.

Lup-20(29)-en-3-ol (6) Sólido branco amorfo; p.f.: $210-212^{\circ} \mathrm{C}$; IV $\left(\mathrm{cm}^{-1}\right)$ : $\mathrm{v}_{\max } 3393$; 2922; 2853; 1381; RMN de ${ }^{13} \mathrm{C}(125 \mathrm{MHz}$, $\left.\mathrm{CDCl}_{3}\right) \delta_{c}: 14,7\left(\mathrm{CH}_{3}-27\right) ; 15,5\left(\mathrm{CH}_{3}-24\right) ; 16,2$ $\left(\mathrm{CH}_{3}-26\right) ; 16,3\left(\mathrm{CH}_{3}-25\right) ; 18,2\left(\mathrm{CH}_{3}-28\right) ; 18,5$ $\left(\mathrm{CH}_{2}-6\right) ; 19,5\left(\mathrm{CH}_{3}-30\right) ; 21,2\left(\mathrm{CH}_{2}-11\right) ; 25,4$ $\left(\mathrm{CH}_{2}-12\right) ; 27,6\left(\mathrm{CH}_{2}-2\right) ; 27,7\left(\mathrm{CH}_{2}-15\right) ; 28,2$ $\left(\mathrm{CH}_{3}-23\right) ; 30,1\left(\mathrm{CH}_{3}-21\right) ; 34,5\left(\mathrm{CH}_{2}-7\right) ; 35,8$ $\left(\mathrm{CH}_{2}-16\right) ; 37,4$ (C-10); 38,3 (C-13); 38,9 $\left(\mathrm{CH}_{2-}^{-}\right.$ 1); 39,1 (C-4); 40,2 ( $\left.\mathrm{CH}_{2}-22\right) ; 41,1$ (C-8); 43,1 (C-14); 43,2 (C-17); 48,2 (CH-19); 48,5 (CH18); 50,7 (CH-9); 55,5 (CH-5); 79,2 (CH-3); $109,5\left(\mathrm{CH}_{2}-29\right) ; 151,2$ (C-20); EM $\mathrm{m} / \mathrm{z} 426$, $\mathrm{C}_{30} \mathrm{H}_{50} \mathrm{O}$.

\subsection{Teste de atividade antioxidante}

O extrato etanólico da própolis vermelha (EEPV) e suas frações (FHEEPV, FDEEPV, FAEEPV e FMEEPV) foram submetidos ao teste de atividade antioxidante. A metodologia utilizada no teste foi a do sequestro de radicais livres, semelhante à descrita por Hegazi e El Hady, (2002), ${ }^{9}$ onde o radical utilizado foi o DPPH (2,2-difenil-1picrilidrazila) na concentração de $60 \mu \mathrm{mol} . \mathrm{L}^{-1}$. Amostras nas concentrações de 0,$001 ; 0,005$; 0,$01 ; 0,05 ; 0,1$ e $1,0 \mathrm{mg} / \mathrm{mL}$ foram dissolvidas em metanol e $1,0 \mathrm{~mL}$ de cada amostra foi adicionada a uma solução metanólica de DPPH (1,0 mL), na concentração de 60 $\mu \mathrm{mol} . \mathrm{L}^{-1}$. Foram realizadas medidas de absorbância na faixa de 520 nm em espectrofotômetro de UV-VIS Cary 50 Conc da Varian, após $30 \mathrm{~min}$. A porcentagem de inibição foi obtida por comparação da 
absorção da solução contendo amostra, em relação a uma solução controle de DPPH sem amostra.

Porcentagem de inibição $=(1-$ Abs. $d a$ amostra/Abs. do DPPH) $\times 100$

Após o cálculo, foi construído um gráfico de porcentagem de inibição versus a concentração. Para o cálculo da $\mathrm{Cl}_{50}$ foi utilizada a equação da reta, substituindo o valor de y por 50 para obtenção da concentração da amostra com capacidade de reduzir $50 \%$ do DPPH.

$\mathrm{O}$ teste foi realizado em triplicata. Como padrões positivos de referência utilizaram-se Trolox ${ }^{\circledR} \quad$ (ácido 6-hidroxi-2,5,7,8tetrametilcroman-2-carboxilico) e Vitamina $C$ (ácido ascórbico), adquiridos da Sigma Aldrich.

\subsection{Ensaio para inibição da enzima acetilcolinesterase}

O extrato etanólico da própolis vermelha (EEPV) e suas frações (FHEEPV, FDEEPV e FAEEPV) foram submetidos ao ensaio colorimétrico qualitativo para inibição da enzima acetilcolinesterase. Este ensaio é baseado no procedimento descrito por Ellman et al. (1961), ${ }^{10}$ e adaptado para CCD por Rhee et al. (2001). ${ }^{11}$

Uma alíquota de $5 \mu \mathrm{L}$ das amostras na concentração de $10 \mathrm{mg} / \mathrm{mL}$ foram aplicados em CCD, borrifados com uma mistura de (1:1) de iodeto de acetilcolina (ACTI) $1 \mathrm{mmol} / \mathrm{L}$ com o reagente de Ellman (ácido 5,5'ditiobis-(2-nitrobenzoico) (DTNB) $1 \mathrm{mmol} / \mathrm{L}$, e depois com a enzima acetilcolinesterase (3 $\mathrm{U} / \mathrm{mL}$ ). Passados $10 \mathrm{~min}$, observa-se o surgimento de uma coloração amarela, porém, onde ocorre a inibição da enzima, aparece um halo branco em torno dos "spots" onde foram aplicadas as amostras. Como controle positivo foi utilizado uma solução do padrão sal de Eserina $(2 \mathrm{mg} / \mathrm{mL})$, e como controle negativo foram utilizados solventes.

\section{Resultados e Discussão}

A investigação fitoquímica do extrato etanólico da própolis vermelha resultou no isolamento e identificação de: 2'-hidroxi-4',7dimetoxiisoflavana (1), 2',7-dihidroxi-4'metoxiisoflavana (2), 2', 4'-dihidroxi-7metoxiisoflavana (3), 4',7-dihidroxi-2'metoxiisoflavana (4), 2', $\quad 4^{\prime}, 4$ trihidroxichalcona (5) e lup-20(29)-en-3-ol (6) (Figura 1). As estruturas destes metabólitos foram elucidadas através de dados espectroscópicos de IV, RMN de ${ }^{1} \mathrm{H}, \mathrm{RMN}$ de ${ }^{13} \mathrm{C}$ (BB e DEPT $135^{\circ}$ ), COSY, HSQC e HMBC e de massas obtidos através do CG-EM.

O espectro de massas de baixa resolução da substância 1 forneceu 0 pico correspondente ao íon molecular em $\mathrm{m} / \mathrm{z} 286$ $\mathrm{Da}$, confirmando a fórmula molecular $\mathrm{C}_{17} \mathrm{H}_{18} \mathrm{O}_{4}$. O espectro de RMN de ${ }^{13} \mathrm{C}-\mathrm{BB}$ apresentou sinais correspondentes a seis carbonos não hidrogenados [todos $\mathrm{sp}^{2}$, inclusive quatro oxigenados em $\delta_{C}$ 154,5 (C2'), 155,4 (C-9), 159,4 (C-7) e 159,7 (C-4')], sete metínicos (seis $\mathrm{sp}^{2}$ e um $\mathrm{sp}^{3}$ ), dois metilênicos (um oxigenado em $\delta_{c} 70,2$ ) e duas metoxilas (Tabela 1).

$\mathrm{O}$ espectro de RMN de ${ }^{1} \mathrm{H}$ de 1 apresentou quatro dupletos na região de sistemas aromáticos em: $\delta_{\mathrm{H}} 7,03\left(\mathrm{~J}=8,7 \mathrm{~Hz}, \mathrm{H}-6^{\prime}\right), 7,0$ $(J=8,6 \mathrm{~Hz}, \mathrm{H}-5), 6,45\left(\mathrm{H}-3^{\prime}\right)$ e $6,37(\mathrm{H}-8)$, referentes a dois acoplamentos orto e dois meta, respectivamente, e dois duplo dupletos em $\delta_{\mathrm{H}} 6,48(J=8,4$ e $2,1 \mathrm{~Hz}, \mathrm{H}-6$ e H$\left.5^{\prime}\right)$. Estas deduções, juntamente com a análise dos espectros de COSY, HSQC e HMBC permitiram identificar a substância como 2'hidroxi-4',7-dimetoxiisoflavana reportada na própolis vermelha. ${ }^{12}$

(1), já

O espectro de massas de baixa resolução da substância 2 forneceu 0 pico correspondente ao íon molecular em $\mathrm{m} / \mathrm{z} 272$ $\mathrm{Da}$, condizente com a fórmula molecular $\mathrm{C}_{16} \mathrm{H}_{16} \mathrm{O}_{4}$. O espectro de $\mathrm{RMN}$ de ${ }^{13} \mathrm{C}-\mathrm{BB}$ apresentou sinais correspondentes a átomos de carbonos oxigenados em $\delta_{c} 159,6$ (C-4'), 
$\delta_{\mathrm{C}} 155,4(\mathrm{C}-7), \delta_{\mathrm{c}} 155,1(\mathrm{C}-9)$ e $\delta_{\mathrm{c}} 154,5$ (C-2') (Tabela 1).

A análise do espectro de $\mathrm{RMN}$ de ${ }^{1} \mathrm{H}$ mostrou absorções na região de hidrogênios aromáticos em $\delta_{\mathrm{H}} 7,02\left(\mathrm{~d}, J=8,4 \mathrm{~Hz}, \mathrm{H}-6^{\prime}\right), \delta_{\mathrm{H}}$ $6,93(\mathrm{~d}, J=8,1 \mathrm{~Hz}, \mathrm{H}-5), \delta_{\mathrm{H}} 6,48(\mathrm{dd}, J=8,4 \mathrm{e}$ $2,3 \mathrm{~Hz}, \mathrm{H}-5^{\prime}$ ), $\delta_{\mathrm{H}} 6,40$ (dd, $J=8,1$ e $2,3 \mathrm{~Hz}, \mathrm{H}-6$ ), $\delta_{H} 6,38\left(d, J=2,3 \mathrm{~Hz}, \mathrm{H}-3^{\prime}\right.$ ) e $\delta_{H} 6,37$ (d, $J=2,3$ $\mathrm{Hz}, \mathrm{H}-8)$, indicando a presença de dois anéis 1,2,4-trissubstituídos. Com base nas informações espectrais apresentadas e com a análise dos espectros de COSY, HSQC e HMBC possibilitou identificar a substância como sendo 2',7-dihidroxi-4'-metoxiisoflavana (2), já relatada na própolis vermelha. ${ }^{13}$

O composto (3) mostrou o pico do íon molecular em $m / z 272$ Da no espectro de massas, que em combinação com os dados de RMN de ${ }^{1} \mathrm{H}$ e ${ }^{13} \mathrm{C}$ permitiu a dedução da fórmula molecular $\mathrm{C}_{16} \mathrm{H}_{16} \mathrm{O}_{4}$. O espectro na região do infravermelho apresentou banda em $3350 \mathrm{~cm}^{-1}$ compatível com a presença de hidroxila fenólica.

O padrão de hidrogenação foi obtido por comparação com o espectro de RMN de ${ }^{13} \mathrm{C}$ DEPT $135^{\circ}$, o qual evidenciou a presença de dois carbonos metilênicos, onde um é oxigenado $\left[\delta_{C} 71,4(C-2)\right]$, e sete carbonos metínicos. Os sinais restantes são referentes a carbonos não hidrogenados (Tabela 1).

A análise do espectro de $\mathrm{RMN}$ de ${ }^{1} \mathrm{H}$ mostrou absorções na região de hidrogênios aromáticos em $\delta_{\mathrm{H}} 6,94(\mathrm{~d}, J=8,3 \mathrm{~Hz}, \mathrm{H}-5), \delta_{\mathrm{H}}$ $6,86\left(\mathrm{~d}, J=8,3 \mathrm{~Hz}, \mathrm{H}-6^{\prime}\right), \delta_{\mathrm{H}} 6,42(\mathrm{dd}, J=8,3$ e $2,4 \mathrm{~Hz}, \mathrm{H}-6), \delta_{\mathrm{H}} 6,33(\mathrm{~d}, J=2,2 \mathrm{~Hz}, \mathrm{H}-8), \delta_{\mathrm{H}}$ $6,32\left(\mathrm{~d}, J=2,4 \mathrm{~Hz}, \mathrm{H}-3^{\prime}\right)$ e $\delta_{\mathrm{H}} 6,26(\mathrm{dd}, J=8,3$ e $2,2 \mathrm{~Hz}, \mathrm{H}-5^{\prime}$ ), indicando a presença de dois anéis 1,2,4-trissubstituídos. Baseando-se em todos os dados espectrais obtidos e com a análise dos espectros de COSY, HSQC e HMBC verificou-se que a substância tratava-se da $2^{\prime}, 4^{\prime}$-dihidroxi-7-metoxiisoflavana (3). ${ }^{12}$

O espectro de absorção na região do IV de (4) revelou sinais bastante semelhantes ao espectro de (1), portanto, sugerimos que a substância (4) pertence à mesma classe da substância (1), ou seja, uma isoflavana.
O espectro de massas de baixa resolução de (4) forneceu o pico em m/z 272 Da correspondente ao íon molecular, confirmando a presença de dois anéis aromáticos e uma estrutura cíclica, condizente com a fórmula molecular $\mathrm{C}_{16} \mathrm{H}_{16} \mathrm{O}_{4}$. Uma comparação feita entre os espectros de RMN de ${ }^{13} \mathrm{C}$ e DEPT $135^{\circ}$ evidenciou a presença de dois carbonos metilênicos, onde um é oxigenado $\left[\delta_{c} 71,2\right.$ (C-2)], sete carbonos metínicos, e um sinal de carbono metílico de metoxila $\left(-\mathrm{OCH}_{3}\right)$ em $\delta_{\mathrm{C}}$ 55,7 (Tabela 1).

A análise do espectro de RMN de ${ }^{1} \mathrm{H}$ de (4) mostrou absorções na região de hidrogênios aromáticos em $\delta_{\mathrm{H}} 6,93\left(\mathrm{~d}, J=8,4 \mathrm{~Hz}, \mathrm{H}-6^{\prime}\right), \delta_{\mathrm{H}}$ $6,84(\mathrm{~d}, J=8,2 \mathrm{~Hz}, \mathrm{H}-5), \delta_{\mathrm{H}} 6,39(\mathrm{~d}, J=2,4 \mathrm{~Hz}$, $\left.H-3^{\prime}\right), \delta_{H} 6,35\left(\mathrm{dd}, J=8,2\right.$ e $\left.2,4 \mathrm{~Hz}, \mathrm{H}-5^{\prime}\right), \delta_{\mathrm{H}}$ $6,31(\mathrm{dd}, J=8,4$ e $2,4 \mathrm{~Hz}, \mathrm{H}-6)$ e $\delta_{\mathrm{H}} 6,24$ (d, $J=$ $2,4 \mathrm{~Hz}, \mathrm{H}-8)$, indicando a presença de dois anéis 1,2,4-trissubstituídos. Estas deduções, juntamente com a análise dos espectros de COSY, HSQC e HMBC permitiram identificar a substância como 4',7-dihidroxi-2'metoxiisoflavana (4), já reportada na própolis vermelha. ${ }^{14}$

O espectro de absorção na região do IV de (5) apresentou uma banda larga em $3284 \mathrm{~cm}$ 1 , característica de deformação axial de ligação O-H; duas absorções em 1629 e 1513 $\mathrm{cm}^{-1}$ referentes às deformações axiais da ligação $\mathrm{C}=\mathrm{C}$ de sistema aromático; uma banda em $1226 \mathrm{~cm}^{-1}$ associada à deformação axial de ligação C-O.

O espectro de massas de baixa resolução apresentou o pico do íon molecular em $\mathrm{m} / \mathrm{z}$ $256 \mathrm{Da}$ correspondente ao íon molecular, condizente com a fórmula molecular $\mathrm{C}_{15} \mathrm{H}_{12} \mathrm{O}_{4}$.

O padrão de hidrogenação de (5) foi obtido por comparação dos espectros de RMN de ${ }^{13} \mathrm{C}$ e DEPT $135^{\circ}$, o qual evidenciou a presença de nove carbonos metínicos, e os sinais restantes referentes a carbonos não hidrogenados.

A análise do espectro de $\mathrm{RMN}$ de ${ }^{1} \mathrm{H}$ mostrou absorções na região de hidrogênios aromáticos em $\delta_{\mathrm{H}} 7,94\left(\mathrm{~d}, J=8,9 \mathrm{~Hz}, \mathrm{H}-6^{\prime}\right), \delta_{\mathrm{H}}$ $7,60\left(\mathrm{~d}, J=8,6 \mathrm{~Hz}, \mathrm{H}-2\right.$ e H-6), $\delta_{\mathrm{H}} 6,84$ (d, $J=$ 
$8,6 \mathrm{~Hz}, \mathrm{H}-3$ e H-5), $\delta_{\mathrm{H}} 6,40$ (dd, $J=8,9$ e $2,3 \mathrm{~Hz}$, $\left.H-5^{\prime}\right)$ e $\delta_{H} 6,28\left(d, J=2,3 \mathrm{~Hz}, \mathrm{H}-3^{\prime}\right)$, indicando a presença de dois anéis, o primeiro 1,2,4trissubstituído e outro 1,4-dissubstituído. Mostrou também deslocamentos químicos em $\delta_{H} 7,77(d, J=15,4 \mathrm{~Hz}, \mathrm{H}-7)$ e $\delta_{H} 7,59(\mathrm{~d}, J=$ $15,4 \mathrm{~Hz}, \mathrm{H}-8)$, característico de um sistema insaturado semelhante ao esqueleto de uma chalcona. Através dos dados espectrais<smiles>COc1ccc(C2COc3cc(OC)ccc3C2)c(O)c1</smiles><smiles>COc1ccc2c(c1)OCC(c1ccc(O)cc1O)C2</smiles><smiles>O=C(/C=C/c1ccc(O)cc1)c1ccc(O)cc1O</smiles>

apresentados juntamente com a análise dos espectros de COSY, HSQC e HMBC, pode-se identificar a substância como sendo $2^{\prime}, 4^{\prime}, 4$ trihidroxichalcona (5), já reportada na própolis vermelha. ${ }^{12}$

O triterpeno lup-20(29)-en-3-ol (6) foi identificado através da análise de dados fornecidos por métodos espectroscópicos e comparação com a literatura. ${ }^{15}$<smiles>COc1ccc(C2COc3cc(O)ccc3C2)c(O)c1</smiles>

2<smiles>COc1cc(O)ccc1C1COc2cc(O)ccc2C1</smiles>

4

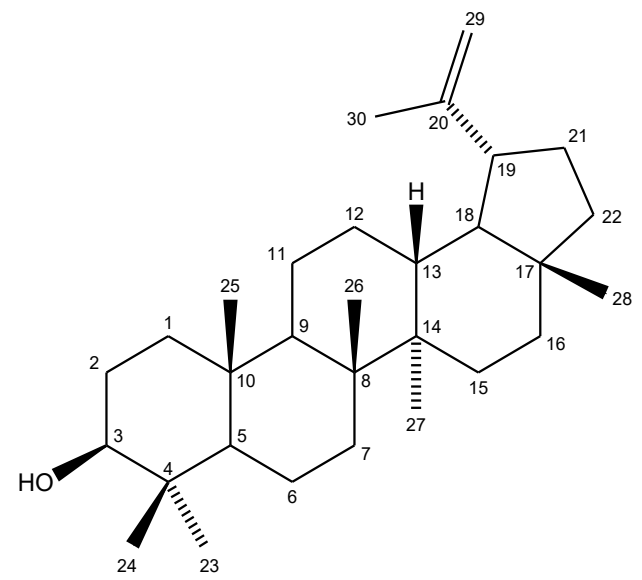

Figura 1. Estruturas dos compostos isolados da própolis vermelha 
Tabela 1. Deslocamentos químicos $(\delta)$ de $R M N$ de ${ }^{13} \mathrm{C}$ das substâncias 1, 2, 3 e 4

\begin{tabular}{c|c|c|c|c}
\hline \multirow{2}{*}{$\mathbf{C}$} & $\mathbf{1}$ & $\mathbf{2}$ & $\mathbf{3}$ & $\mathbf{4}$ \\
\cline { 2 - 5 } & $\delta_{\mathrm{c}}$ & $\delta_{\mathrm{c}}$ & $\delta_{\mathrm{c}}$ & $\delta_{\mathrm{c}}$ \\
\hline $\mathbf{2}$ & 70,2 & 70,1 & 71,4 & 71,2 \\
$\mathbf{3}$ & 32,1 & 31,9 & 33,2 & 33,2 \\
$\mathbf{4}$ & 30,6 & 30,6 & 31,5 & 31,5 \\
$\mathbf{5}$ & 130,4 & 130,6 & 131,3 & 131,4 \\
$\mathbf{6}$ & 106,4 & 108,1 & 108,0 & 109,1 \\
$\mathbf{7}$ & 159,4 & 155,4 & 160,6 & 157,3 \\
$\mathbf{7 a}$ & 55,6 & - & 55,8 & - \\
$\mathbf{8}$ & 102,4 & 103,4 & 102,4 & 103,9 \\
$\mathbf{9}$ & 155,4 & 155,1 & 156,6 & 156,5 \\
$\mathbf{1 0}$ & 120,2 & 114,9 & 116,2 & 115,1 \\
$\mathbf{1}$ & 114,7 & 120,1 & 120,1 & 121,5 \\
$\mathbf{2}$ & 154,4 & 154,5 & 157,4 & 160,9 \\
$\mathbf{2}^{\prime} \mathbf{a}$ & - & - & 103,7 & 55,7 \\
$\mathbf{3}^{\prime}$ & 101,7 & 102,4 & 103,7 & 102,6 \\
$\mathbf{4}$ & 159,7 & 159,6 & 158,1 & 157,6 \\
$\mathbf{4} \mathbf{a}$ & 55,6 & 55,6 & - & - \\
$\mathbf{5}$ & 107,5 & 106,2 & 107,8 & 105,9 \\
$\mathbf{6}^{\prime}$ & 128,5 & 128,4 & 129,0 & 129,0 \\
\hline
\end{tabular}

O extrato etanólico da própolis vermelha (EEPV) e suas frações (FHEEPV, FDEEPV, FAEEPV e FMEEPV) foram submetidos ao teste de atividade antioxidante utilizando a metodologia do sequestro do radical $\mathrm{DPPH} .{ }^{9}$ Foi observada atividade significativa na concentração de $1 \mathrm{mg} / \mathrm{mL}$ para o extrato (EEPV) e para as frações acetato de etila (FAEEPV) e metanólica (FMEEPV), com inibição de 99,8\%. A Tabela 2 mostra os resultados obtidos no ensaio de atividade antioxidante, seus valores de $\mathrm{Cl}_{50}$ e dados da literatura. Analisando os dados, vemos que tanto o extrato quanto suas frações (FAEEPV e FMEEPV) obtiveram resultados de $\mathrm{Cl}_{50}$ significativos e superior ao padrão Vitamina C. A literatura reporta atividade antioxidante de uma amostra de própolis coletada em Marechal Deodoro - AL, usando a mesma metodologia, entretanto, os dados reportados apresentam valores inferiores.

Tabela 2. Resultado do teste de atividade antioxidante

\begin{tabular}{|c|c|c|c|c|}
\hline \multirow[t]{2}{*}{ Amostras } & \multicolumn{2}{|c|}{ Concentração (mg/mL) } & \multirow[t]{2}{*}{$\mathrm{Cl}_{50}(\mathrm{mg} / \mathrm{mL})$} & \multirow[t]{2}{*}{ Literatura $^{1}$} \\
\hline & $0,1 \mathrm{mg} / \mathrm{mL}$ & $1 \mathrm{mg} / \mathrm{mL}$ & & \\
\hline EEPV & $97,2 \%$ & $99,8 \%$ & $2,4 \cdot 10^{-2} \pm 6,4 \cdot 10^{-3}$ & $50,5 \%$ \\
\hline FHEEPV & NA & NA & NA & $74,4 \%$ \\
\hline FDEEPV & $24,5 \%$ & $48,3 \%$ & - & $49,8 \%$ \\
\hline FAEEPV & $95,8 \%$ & $99,8 \%$ & $2,8 \cdot 10^{-2} \pm 3,4 \cdot 10^{-3}$ & - \\
\hline FMEEPV & $94,8 \%$ & $99,8 \%$ & $3,2 \cdot 10^{-2} \pm 1,2 \cdot 10^{-3}$ & - \\
\hline Trolox & $99,8 \%$ & $99,9 \%$ & $2,6.10^{-3} \pm 2,3.10^{-4}$ & - \\
\hline Vitamina C & $92,8 \%$ & $99,8 \%$ & $4,3 \cdot 10^{-2} \pm 1,9 \cdot 10^{-2}$ & - \\
\hline
\end{tabular}

NA - não apresentou atividade

Os resultados da atividade anticolinesterásica estão mostrados na
Tabela 3. Nos resultados obtidos no teste de inibição da enzima acetilcolinesterase, 
observou-se resultados positivos em todas as amostras testadas (EEPV, FDEEPV, FAEEPV e FMEEPV) exceto FHEEPV. Dentre as amostras que apresentaram resultados significativos, merecem destaque a FAEEPV e FMEEPV, os quais apresentaram maiores valores de halos de inibição $(\mathrm{HI})$ da enzima, $0,9 \mathrm{~cm}$, sendo o valor igual ao do controle positivo sal de Eserina.

Tabela 3. Resultado do teste de inibição da atividade da enzima acetilcolinesterase

\begin{tabular}{ccc}
\hline Amostras & HI (cm) & Resultado \\
\hline EEPV & 0,6 & Positivo \\
FHEEPV & -- & Negativo \\
FDEEPV & 0,8 & Positivo \\
FAEEPV & 0,9 & Positivo \\
FMEEPV & 0,9 & Positivo \\
Sal de Eserina & 0,9 & Controle positivo \\
\hline
\end{tabular}

$\mathrm{HI}$ - halo de inibição

\section{Considerações Finais}

As análises espectroscópicas e espectrométricas realizadas permitiram a identificação de seis substâncias isoladas da própolis vermelha [2'-hidroxi-4',7dimetoxiisoflavana (1); 2',7-dihidroxi-4'metoxiisoflavana (2); $\quad 2^{\prime}, 4^{\prime}$-dihidroxi-7metoxiisoflavana (3); 4',7-dihidroxi-2'metoxiisoflavana (4); 2',4',4trihidroxichalcona (5) e do lup-20(29)-en-3-ol (6)], sendo quatro isoflavonas, uma chalcona e um triterpeno.

Extrato e frações da própolis vermelha apresentaram atividade significativa como antioxidante no ensaio do DPPH, e ainda atividade anticolinesterase. Por ser considerado um suplemento alimentar de alto valor nutritivo e tendo na sua composição constituintes químicos como as isoflavanas, pode-se então selecioná-la como potencial fonte de compostos a serem utilizados no tratamento de várias enfermidades entre estas o Alzheimer.

\section{Agradecimentos}

Os autores agradecem as instituições de fomento à pesquisa: CNPq e CAPES pelas bolsas concedidas.

\section{Referências Bibliográficas}

${ }^{1}$ Cabral, I. S. R.; Oldoni, T. L. C.; Prado, A.; Bezerra, R. M. N.; de Alencar, S. M. Composição fenólica, atividade antibacteriana e antioxidante da própolis vermelha brasileira. Química Nova 2009, 32, 1523. [CrossRef]

${ }^{2}$ Cunha, L. C.; Alves, L. D. S.; Santana, L. C. L. R.; Nunes, G. B. L.; Rolim Neto, P. J. A própolis no combate a tripanossomatídeos de importância médica: uma perspectiva terapêutica para doença de chagas e leishmaniose. Revista de Patologia Tropical 2011, 40, 105. [CrossRef]

${ }^{3}$ Frozza, C. O. S.; Garcia, C. S. C.; Gambato, G.; de Souza, M. D. O.; Salvador, M.; Moura, S.; Padilha, F. F.; Seixas, F. K.; Collares, T.; Borsuk, S.; Dellagostin, O. A.; Henriques, J. A. P.; Roesch-ely, M. Chemical characterization, antioxidant and cytotoxic activities of Brazilian red propolis. Food and Chemical Toxicology 2013, 52, 137. [CrossRef]

${ }^{4}$ Alencar, S. M.; Oldoni, T. L. C.; Castro, M. L.; Cabral, I. S. R.; Costa-Neto, C. M.; Cury, J. A.; Rosalen, P. L.; Ikegaki, M. Chemical 
composition and biological activity of a new type of Brazilian propolis: Red propolis. Journal of Ethnopharmacology 2007, 113, 278. [CrossRef]

${ }^{5}$ Kamiya, T.; Nishihara, H.; Hara, H.; Adachi, T. Ethanol Extract of Brazilian Red Propolis Induces Apoptosis in Human Breast Cancer MCF-7 Cells Through Endoplasmic Reticulum Stress. Journal of Agricultural and Food Chemistry 2012, 60, 11065. [CrossRef]

${ }^{6}$ Oldoni, T. L. C.; Cabral, I. S. R.; D'arce, M. A. B. R.; Rosalen, P. L.; Ikegaki, M.; Nascimento, A. M.; Alencar, S. M. Isolation and analysis of bioactive isoflavonoids and chalcone from a new type of Brazilian propolis. Separation and Purification Technology 2011, 77, 208. [CrossRef]

${ }^{7}$ Li, F.; Awale, S.; Tezuka, Y.; Kadota, S. Cytotoxic constituents from Brazilian red propolis and their structure-activity relationship. Bioorganic \& Medicinal Chemistry 2008, 16, 5434. [CrossRef]

${ }^{8}$ Bueno-Silva, B.; Alencar, S. M.; Koo, H.; Ikegaki, M.; Silva, G. V. J.; Napimoga, M. H.; Rosalen, P. L. Anti-Inflammatory and Antimicrobial Evaluation of Neovestitol and Vestitol Isolated from Brazilian Red Propolis. Journal of Agricultural and Food Chemistry 2013, 61, 4546. [CrossRef]

${ }^{9}$ Hegazi, A. G.; El Hady, F. K. A. Egyptian Propolis: 3. Antioxidant, Antimicrobial
Activities and Chemical Composition of Propolis from Reclaimed Lands. Zeitschrift für Naturforschung 2002, 57c, 395. [Link]

${ }^{10}$ Ellman, G. L.; Courtney, K. D.; Andres Jr., V.; Featherstone, R. M. A new and rapid colorimetric determination of acetylcholinesterase activity. Biochemical Pharmacology 1961, 7, 88. [CrossRef]

${ }^{11}$ Rhee, I. K.; de Meent, M. V.; Ingkaninan, K.; Verpoorte, R. Screening for acetylcholinesterase inhibitors from Amaryllidaceae using silica gel thin-layer chromatography in combination with bioactivity staining. Journal of Chromatography A 2001, 915, 217. [CrossRef] 12 Fernández, M. C.; Tese de Doutorado, Universidad de La Habana, 2007. [Link]

${ }^{13}$ Piccinelli, A. L.; Fernandez, M. C.; CuestaRubio, O.; Hernández, I. M.; de Simone, F.; Rastrelli, L. Isoflavonoids Isolated from Cuban Propolis. Journal of Agricultural and Food Chemistry 2005, 53, 9010. [CrossRef]

${ }^{14}$ Hasan, N.; Osman, H.; Mohamad, S.; Chong, W. K.; Awang, K.; Zahariluddin, A. S. M. The chemical components of Sesbania grandiflora root and their antituberculosis activity. Pharmaceuticals 2012, 5, 882. [CrossRef]

${ }^{15}$ Albuquerque, I. L.; Tese de Doutorado, Universidade Federal do Ceará, 2007. [Link] 